\title{
e-MERLIN and VLBI observations of the luminous infrared galaxy IC 883: a nuclear starburst and an AGN candidate revealed
}

\author{
C. Romero-Cañizales ${ }^{1,2}$, M. A. Pérez-Torres ${ }^{1}$, A. Alberdi ${ }^{1}$, M. K. Argo ${ }^{3,4}$, R. J. Beswick ${ }^{4}$, E. Kankare ${ }^{2}$, F. Batejat ${ }^{5}$,
} A. Efstathiou ${ }^{6}$, S. Mattila ${ }^{2}$, J. E. Conway ${ }^{5}$, S. T. Garrington ${ }^{4}$, T. W. B. Muxlow ${ }^{4}$, S. D. Ryder ${ }^{7}$, and P. Väisänen ${ }^{8}$

1 Instituto de Astrofísica de Andalucía - CSIC, PO Box 3004, 18008 Granada, Spain e-mail: crroca@utu.fi

2 Tuorla Observatory, Department of Physics and Astronomy, University of Turku, Väisäläntie 20, 21500 Piikkiö, Finland

3 Netherlands Institute for Radio Astronomy (ASTRON), Postbus 2, 7990 AA Dwingeloo, The Netherlands

${ }^{4}$ Jodrell Bank Centre for Astrophysics, Alan Turing Building, School of Physics and Astronomy, The University of Manchester, Manchester M13 9PL, UK

5 Onsala Space Observatory, 43992 Onsala, Sweden

${ }^{6}$ School of Sciences, European University Cyprus, Diogenes Street, Engomi, 1516 Nicosia, Cyprus

7 Australian Astronomical Observatory, PO Box 296, Epping, 1710 NSW, Australia

8 South African Astronomical Observatory, PO Box 9, 7935 Observatory, South Africa

Received 13 January 2012 / Accepted 10 May 2012

\section{ABSTRACT}

\begin{abstract}
Context. The high star formation rates of luminous infrared galaxies (LIRGs) make them ideal places for core-collapse supernova (CCSN) searches. Massive star formation can often be found in coexistence with an active galactic nucleus (AGN), contributing jointly to the energy source of LIRGs. At radio frequencies, where light is unaffected by dust extinction, it is possible to detect compact components within the innermost LIRG nuclear regions, such as SNe and SN remnants, as well as AGN buried deep in the LIRG nuclei.

Aims. Our study of the LIRG IC 883 aims at: (i) investigating the parsec-scale radio structure of the (circum-)nuclear regions of IC 883; (ii) detecting at radio frequencies the two recently reported circumnuclear SNe 2010cu and 2011hi, which were discovered by near-IR (NIR) adaptive optics observations of IC 883; and (iii) further investigating the nature of SN 2011 hi at NIR wavelengths. Methods. We used the electronic European very long baseline interferometry (VLBI) Network (e-EVN) at $5 \mathrm{GHz}$, and the electronic Multi-Element Remotely Linked Interferometer Network (e-MERLIN) at $6.9 \mathrm{GHz}$, to observe contemporaneously the LIRG IC 883 at high angular-resolution (from tens to hundreds of milliarcsec) and with high sensitivity $(<70 \mu \mathrm{Jy})$, complemented by archival VLBI data at $5 \mathrm{GHz}$ and $8.4 \mathrm{GHz}$. We also used the Gemini North telescope to obtain late-time $J H K$ photometry for SN $2011 \mathrm{hi}$.

Results. The circumnuclear regions traced by e-MERLIN at $6.9 \mathrm{GHz}$ have an extension of $\sim 1 \mathrm{kpc}$, at a position angle of $130^{\circ}$, and show a striking double-sided structure, which very likely corresponds to a warped rotating ring, in agreement with previous studies. Our e-EVN observations at $5 \mathrm{GHz}$ and complementary archival VLBI data at $5 \mathrm{GHz}$ and $8.4 \mathrm{GHz}$, reveal various milliarcsec compact components in the nucleus of IC 883. A single compact source, an AGN candidate, dominates the emission at both nuclear and circumnuclear scales, as imaged with the e-EVN and e-MERLIN, respectively. The other milliarcsec components are strongly indicative of ongoing nuclear CCSN activity. Our e-EVN observations also provided upper limits to the radio luminosity of the two SNe in IC 883 recently discovered at NIR wavelengths. We refine the classification of SN 2011hi as a Type IIP SN according to our latest epoch of Gemini North observations acquired in 2012, in agreement with a low-luminosity radio SN nature. We estimate a CCSN rate lower limit of $1.1_{-0.6}^{+1.3} \mathrm{yr}^{-1}$ for the entire galaxy, based on three nuclear radio SNe and the circumnuclear SNe 2010cu and 2011hi.
\end{abstract}

Key words. galaxies: starburst - galaxies: individual: IC 883 - radio lines: stars - radiation mechanisms: non-thermal

\section{Introduction}

The excess of radio continuum and infrared (IR) emission in luminous IR galaxies (LIRGs) is associated with the vigorous formation of massive stars $\left(M \gtrsim 8 M_{\odot}\right)$ and/or an active galactic nucleus (AGN) (see e.g., Sanders \& Mirabel 1996). Corecollapse supernovae (CCSNe) serve as probes of the star formation rate (SFR) of massive stars, hence their detection in LIRGs is a pertinent task.

IC 883 is a LIRG with an IR luminosity $\left(L_{\mathrm{IR}}=\right.$ $L[8-1000 \mu \mathrm{m}])$ of about $4.7 \times 10^{11} L_{\odot}$ at a distance of $100 \mathrm{Mpc}$ ( 1 mas $\approx 0.48 \mathrm{pc}$ ), according to Sanders et al. (2003). This LIRG is probably the result of the merger of two disc galaxies (Smith et al. 1995), as suggested by its associated tidal tails, a diffuse one approximately perpendicular to the region where gas and dust preferentially concentrate (i.e., the disc) as seen in optical and near-IR (NIR) studies (e.g., Smith et al. 1995; Scoville et al. 2000; Modica et al. 2012), and a narrow one along the disc itself (Keel 1985).

Taking $L_{\mathrm{IR}}$ as a measure of the rate at which massive stars are formed, results in a corresponding CCSN rate, $v_{\mathrm{SN}}$, of $\sim 1.3 \mathrm{yr}^{-1}$ for IC 883 (assuming the empirical relation obtained by Mattila \& Meikle 2001). The optical spectroscopic studies of Veilleux et al. (1995) led to the classification of the IC 883 nucleus as a low-ionisation narrow emission-line region. However, Yuan et al. (2010) reclassified this galaxy as a composite starburstAGN, based on the revised optical diagnostic diagrams of Kewley et al. (2006). The multi-wavelength study of IC 883 by Modica et al. (2012) also provides evidence of its composite nature. It is consequently expected that an AGN contributes to 
Table 1. Observational parameters of the radio observations.

\begin{tabular}{|c|c|c|c|c|c|c|c|c|c|}
\hline $\begin{array}{l}\text { Epoch } \\
\text { label }\end{array}$ & Project & Array & $\begin{array}{c}\text { Observing } \\
\text { date }\end{array}$ & $\begin{array}{c}v \\
(\mathrm{GHz})\end{array}$ & $\begin{array}{c}\text { Phase } \\
\text { calibrator }\end{array}$ & $\begin{array}{c}P_{v} \\
(\mathrm{Jy} / \text { beam })\end{array}$ & Weighting & $\begin{array}{c}\text { Convolving beam } \\
\text { (mas) }\end{array}$ & $\begin{array}{c}\mathrm{rms} \\
(\mu \mathrm{Jy} / \text { beam })\end{array}$ \\
\hline (1) & (2) & (3) & (4) & (5) & (6) & (7) & (8) & (9) & (10) \\
\hline EM & - & e-MERLIN & 2011-03-19 & 6.9 & $\mathrm{~J} 1324+3622$ & 0.07 & $\mathrm{~N}, 1$ & $165.23 \times 88.35$ at $11.6^{\circ}$ & 44.0 \\
\hline V1 & BN026 & VLBA & 2004-08-13 & 5.0 & $\mathrm{~J} 1317+3425$ & 0.25 & $\mathrm{~N}, 0$ & $3.71 \times 1.47$ at $-0.1^{\circ}$ & 84.7 \\
\hline V2 & BN027 & VLBA & 2004-09-20 & 5.0 & $\mathrm{~J} 1310+3220$ & 1.41 & $\mathrm{~N}, 1$ & $3.48 \times 1.65$ at $17.6^{\circ}$ & 119.0 \\
\hline V3 & BN027 & VLBA & 2005-07-11 & 5.0 & $\mathrm{~J} 1310+3220$ & 1.19 & $\mathrm{~N}, 2$ & $3.43 \times 1.78$ at $11.5^{\circ}$ & 107.0 \\
\hline V4 & EP055 & EVN & 2006-06-15 & 5.0 & $\mathrm{~J} 2333+3901$ & 0.81 & $\mathrm{U}, 1$ & $7.99 \times 6.00$ at $-81.3^{\circ}$ & 162.0 \\
\hline V5 & RR006 & e-EVN & 2011-03-23 & 5.0 & $\mathrm{~J} 1317+3425$ & 0.35 & $\mathrm{U}, 1$ & $9.20 \times 6.36$ at $-76.1^{\circ}$ & 66.2 \\
\hline V6 & BC196 & VLBA & 2011-05-15 & 8.4 & $\mathrm{~J} 2330+3348$ & 0.33 & $\mathrm{~N}, 0$ & $2.41 \times 1.17$ at $-8.3^{\circ}$ & 174.0 \\
\hline
\end{tabular}

Notes. Phase reference sources used in each observation are listed along with their associated peak intensities (Cols. 6 and 7). For the different IC 883 maps, Col. 8 lists the weighting parameters used in the imaging process: $\mathrm{N}=$ natural, $\mathrm{U}=$ uniform, and the number corresponds to the value of the ROBUST parameter). Column 9 lists the resolution, and Col. 10 the attained root mean square (rms) noise.

the total $L_{\mathrm{IR}}$ in IC 883, thus that the $v_{\mathrm{SN}}$ should be lower than $1.3 \mathrm{yr}^{-1}$. At radio frequencies, Smith et al. (1998) and Parra et al. (2010) have found evidence of compact AGN activity based on very long baseline interferometry (VLBI) studies of IC 883.

IC 883 is part of our program "An ALTAIR study of Supernovae in Luminous Infrared Galaxies", using Gemini North with its laser guide star adaptive optics (AO) system (e.g., Kankare et al. 2008). During the four-year survey, our program has yielded the discovery of two CCSNe in IC 883 within a 12-month period: SN 2010cu (24 Feb. 2010, Ryder et al. 2010) and SN 2011hi (11 Feb. 2011, Kankare et al. 2011). Kankare et al. (2012) concluded that either a Type IIP or a IIn/L SN provided the best fits to the NIR light curves of SNe 2010cu and 2011 hi.

In this paper, we report our radio observations towards the nuclear and circumnuclear regions of IC 883, as well as the most recent NIR epoch obtained with Gemini North on 31 January 2012, that was not included in Kankare et al. (2012). In Sect. 2, we give details of our radio and NIR observations, Sect. 3 contains our results and discussion, and Sect. 4 contains the summary of our study.

\section{Observations}

IC 883 was observed at radio frequencies with the electronic Multi-Element Remotely Linked Interferometer Network (e-MERLIN) and the electronic European VLBI Network $\left(\mathrm{e}-\mathrm{EVN}^{1}\right)$, and at NIR wavelengths with the Gemini North telescope. Our observations had been designed to image the parsecscale radio structure of the (circum-)nuclear regions of IC 883, and detect $\mathrm{SNe} 2010 \mathrm{cu}$ and $2011 \mathrm{hi}$ at radio frequencies, as well as to investigate the nature of SN 2011hi at NIR wavelengths. To complement our radio observations, we also analysed publicly available archival VLBI data.

In Table 1, we provide some details of the observational parameters of the different radio observations included in our study: e-MERLIN, e-EVN, and additional Very Long Baseline Array (VLBA) and EVN observations.

\section{1. e-MERLIN observations and data reduction}

We carried out our e-MERLIN observations of IC 883 at $6.9 \mathrm{GHz}$ (median central frequency) on 19 March 2011. These were

\footnotetext{
1 The development of e-VLBI within the EVN has been made possible via EXPReS project funded by the EC FP6 IST Integrated Infrastructure Initiative contract \# 026642 - with a goal of achieving 1 Gbps e-VLBI real-time data transfer and correlation.
}

director's discretionary time (DDT) observations within the commissioning phase of e-MERLIN, which included the $25 \mathrm{~m}$ diameter antennas Mark II, Defford, Knockin, Darnhall, and Pickmere. The observations lasted $\sim 24 \mathrm{~h}$, of which approximately 20 were spent on target and $10 \mathrm{~h}$ were ultimately usable after editing. Four sub-bands (512 channels each) with dual polarisation were used, accounting for a total bandwidth of $512 \mathrm{MHz}$.

We analysed the data within the National Radio Astronomy Observatory (NRAO) Astronomical Image Processing System (AIPS). 3C286 set the absolute flux density scale following an iterative process. The flux estimated for 3C286 on the shortest baseline (Mark II-Pickmere), at the centre of the different sub-bands, was on average 5.56 Jy. We then calibrated the amplitude of DA 193, which is a bright and unresolved source as seen by all e-MERLIN baselines, resulting in an average flux density of 3.72 Jy. Finally, we used DA 193 to set the flux density of the phase reference source, J1324+3622, which resulted in an average flux density of $68.25 \mathrm{mJy}$. We performed a series of phase-only self-calibration iterations of the phase reference source, before phase-calibrating the target source. To account for the correlation offset from the position of the strongest source in the field, we used the task UVSUB in AIPS. To improve the sensitivity, we performed phase-only self-calibration iterations on the target source. We achieved a thermal root mean square (rms) noise in the IC 883 map of $44 \mu \mathrm{Jy} /$ beam, for a beam size of $165 \times 88$ mas at $-11.6^{\circ}$ (see left panel in Fig. 1), which was obtained with natural weighting and ROBUST $=1$ within AIPS.

\section{2. e-EVN observations and data reduction}

We observed IC 883 on 23 March 2011 (ToO project: RR006, P.I.: Romero-Cañizales) at $5 \mathrm{GHz}$, with the e-EVN, which included the following antennas (diameter, location): Effelsberg (100 m, Germany), Mark II (25 m, UK), Medicina (32 m, Italy), Onsala (25 m, Sweden), Torun (32 m, Poland), Westerbork array $(14 \times 25 \mathrm{~m}, \mathrm{NL})$ and Yebes (40 m, Spain).

RR006 was a two-hour experiment $(\sim 1.3 \mathrm{~h}$, total time on source), recorded at $1024 \mathrm{Mbps}$ using eight sub-bands, each of $16 \mathrm{MHz}$ and dual polarisation. The data were correlated at the EVN MkIV data processor at JIVE with an averaging time of $2 \mathrm{~s}$. The point-like source $\mathrm{J} 1159+2914(2.45 \mathrm{Jy}$ at $5 \mathrm{GHz})$ was used as a fringe finder and bandpass calibrator. J1317+3425 (0.35 Jy at $5 \mathrm{GHz}$ ), at $\sim 0.7^{\circ}$ angular distance from IC 883 , served as a phase reference source. We alternated $3.4 \mathrm{~min}$ scans of IC 883 with 1.2 min scans of $\mathrm{J} 1317+3425$. The data were analysed with AIPs. We also used the Caltech program DIFMAP (Shepherd 
C. Romero-Cañizales et al.: IC 883 revealed by e-MERLIN and VLBI observations

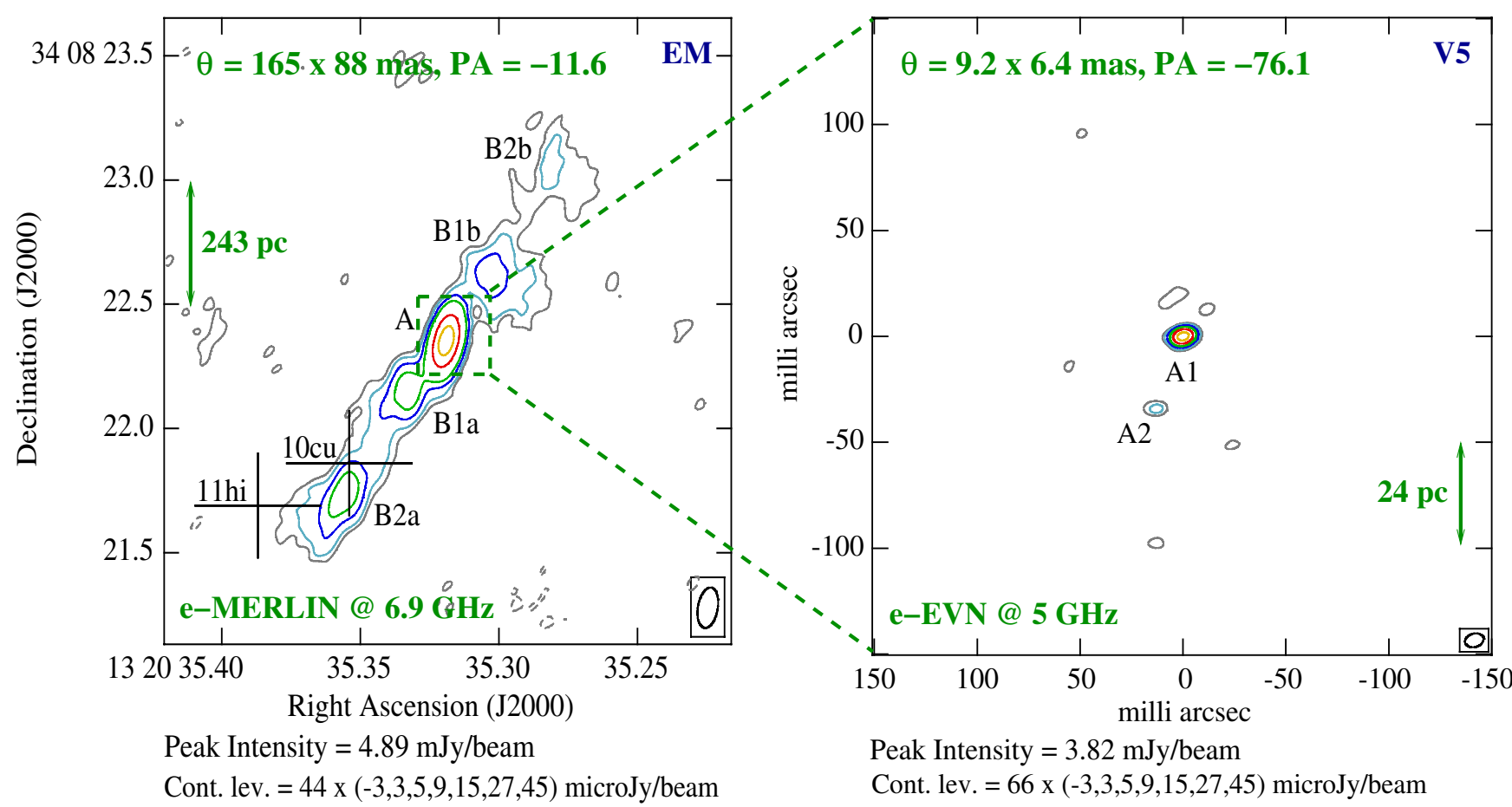

Fig. 1. IC 883 contour images at a median central frequency of $6.9 \mathrm{GHz}$ obtained with e-MERLIN (left), and at $5 \mathrm{GHz}$ with the e-EVN (right), in observations carried out in March 2011. The rms noise in the two images is 44 and $66 \mu \mathrm{Jy} / \mathrm{beam}$, respectively, and the convolving beam in each case is indicated in the upper left corner of the maps. Dashed contours represent $-3 \sigma$ levels. Note that the brightest component on e-MERLIN scales is dominated by a compact source seen on mas scales with the e-EVN. The crosses in the left panel indicate the coordinates and positional errors of SNe 2010cu and 2011hi, according to the values reported in Kankare et al. (2012).

et al. 1995) to image the calibrators and assess the performance of each antenna.

In an attempt to detect a radio counterpart to SN2011hi, its coordinates derived from the NIR images were used for pointing and correlation. A local $\mathrm{rms}$ of $\sim 30 \mu \mathrm{Jy} /$ beam was achieved at the pointing centre. Since no radio source was detected above $3 \sigma$ at that position, we corrected the visibility data with the task UVSUB in AIPS, as we did for the e-MERLIN data, to account for the correlation offset from the position of the strongest source in the field, i.e., to the radio nucleus of IC 883. The shift of $\sim 0.8$ arcsec is well within the primary beam of each antenna in the used array.

For the imaging process within AIPS, we used different weighting schemes in order to test the reliability of our results. Owing to the scarce uv-coverage, the use of natural weighting tends to give rise to secondary side lobes at the 60 per cent level of the main lobe, which can produce artifacts in the reconstruction imaging process that can be confused with putative sources. Our final image was made using uniform weighting and ROBUST $=1$. We achieved a final thermal $\mathrm{rms}$ in the map of $66.2 \mu \mathrm{Jy} /$ beam, for a beam size of $9.20 \times 6.36$ mas at $-76.1^{\circ}$ (see Fig. 1, right panel).

\subsection{Archival VLBI data}

We analysed VLBA data from projects BN026 and BN027 at $5 \mathrm{GHz}$, and BC196 at $8.4 \mathrm{GHz}$. The VLBA observations under program BC196 were processed with the DiFX VLBI correlator (Deller et al. 2011). We also report EVN observations at 5 GHz made on 15 June 2006 (project: EP055, P.I.: R. Parra) see Table 1.

In project EP055, IC 883 was observed in four scans spread over $24 \mathrm{~h}$ to optimize the uv-coverage for a total observation time on source of $\sim 1.3 \mathrm{~h}$. The array used comprised $10 \mathrm{EVN}$ stations: Lovell (76 m, UK), Cambridge (32 m, UK), Westerbork array $(14 \times 25 \mathrm{~m}, \mathrm{NL})$, Effelsberg (100 m, Germany), Medicina (32 m, Italy), Noto (32 m, Italy), Onsala (25 m, Sweden), Torun ( $32 \mathrm{~m}$, Poland), Urumqi (25 m, China), and Shanghai (25 m, China). The observations were performed in a $1024 \mathrm{Mbps}$ dual polarization mode. The data were correlated at JIVE in the Netherlands with an averaging time of $2 \mathrm{~s}$.

All the additional VLBI data were edited and reduced using standard procedures within AIPS. The phase reference calibrator used in project BN027 is slightly resolved at VLBA scales, and we thus corrected the fringe solutions from its structure. We did not apply any self-calibration to obtain the final images of IC 883. In the case of project EP055, the data from the two Chinese antennas (Ur and Sh) as well as from Cm could not be properly calibrated, so we omitted these antennas in the imaging process.

As in the case of our e-EVN epoch, we tested the reliability of the sources in the different VLBI maps, by using different weighting schemes. We used the AIPs task UVSUB to apply shifts in $\alpha$ and $\delta$ to the visibility data on all the additional archival VLBI epochs. This allowed us to have all the maps centred at the same position $\left(\alpha(\mathrm{J} 2000)=13^{\mathrm{h}} 20^{\mathrm{m}} 35^{\mathrm{s}} .3184\right.$, $\left.\delta(\mathbf{J} 2000)=34^{\circ} 08^{\prime} 22^{\prime \prime} \cdot 352\right)$. Since the resulting images have a different convolving beam and were thus mapped with different pixel sizes, we changed their geometry to match that of our e-EVN epoch (pixel size of 0.5 mas), using the task OHGEO within AIPS.

\subsection{Gemini North observations}

Our Gemini North observations of IC 883 were conducted with the ALTAIR Laser Guide Star AO system on the Near-InfraRed 


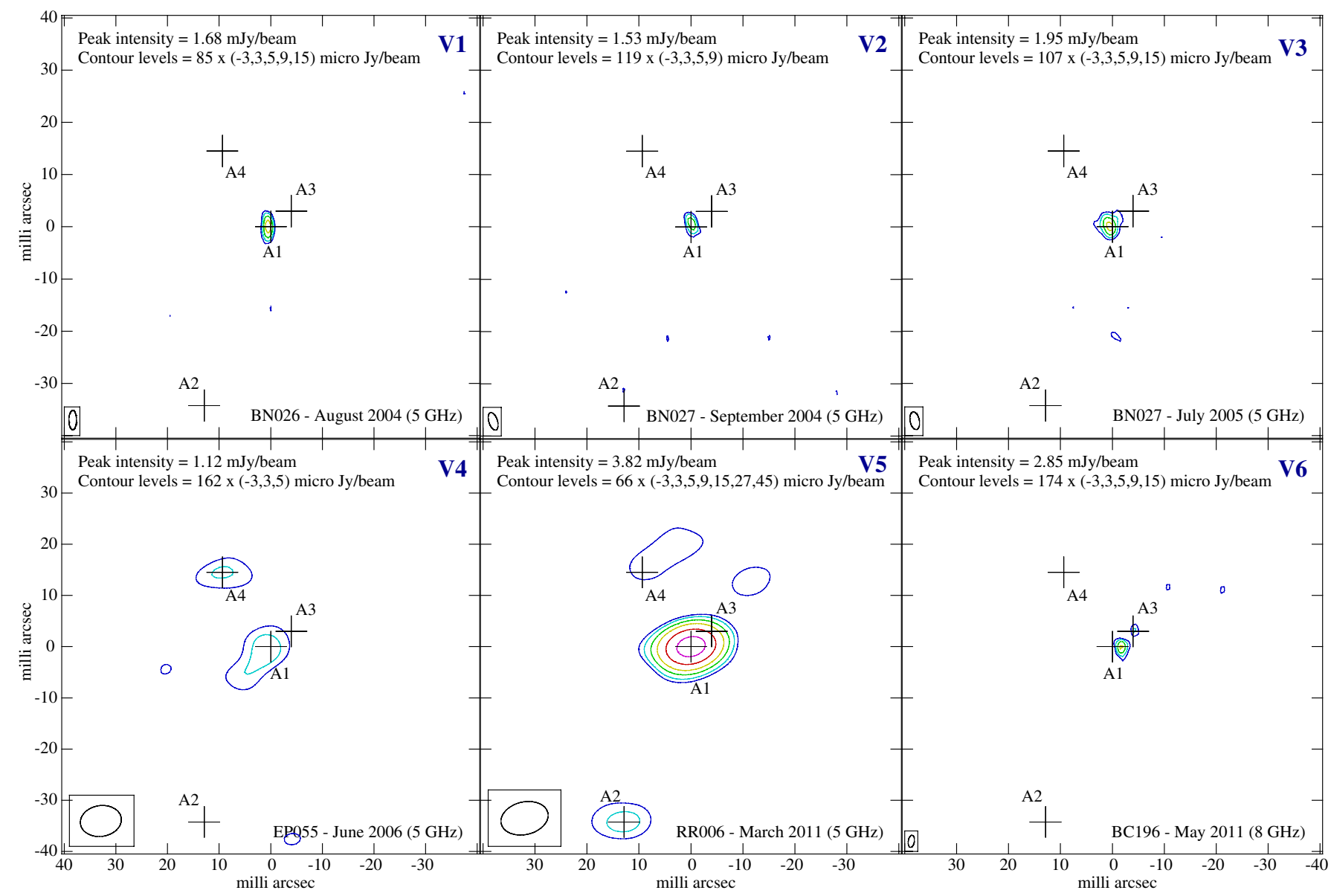

Fig. 2. IC 883 contour maps of the VLBI observations listed in Table 1 . All the maps are centred on $\alpha(\mathrm{J} 2000)=13^{\mathrm{h}} 20^{\mathrm{m}} 35^{\mathrm{s}} \cdot 3184, \delta(\mathrm{J} 2000)=$ $34^{\circ} 08^{\prime} 22^{\prime \prime} 352$. The crosses indicate the positions from the detection epoch of the different $5 \sigma$ sources reported in Table 2: A1 and A2 from experiment V5; A3 from experiment V6; and A4 from experiment V4.

Imager (NIRI). The LIRG was observed in 11 separate epochs between 2008 April 15.5 UT and 2012 January 31.6 UT, covering a period of $3.8 \mathrm{yr}$. Here we report the results from the most recent epoch of $J H K$ imaging obtained in 2012 under program GN-2012A-Q-56 (P.I.: S. Ryder); the previous epochs are reported in Kankare et al. (2012). For details of the data reduction, calibration, image subtraction process, and photometry of the ALTAIR/NIRI data on SN 2010cu and SN 2011hi, we refer to Kankare et al. (2012).

Image subtraction based on the ISIS 2.2 (Alard \& Lupton 1998; Alard 2000) package was used to compare the 2012 JHK images of IC 883 to the reference data obtained on 2010 May 4-5 UT. This revealed SN 2011hi to have faded below the detection limit of the Gemini images. As in our derivation of the $J$-band upper limit for SN 2010cu in Kankare et al. (2012), we used the QUBA package (see Valenti et al. 2011) running standard IRAF tasks, to measure the faintest detectable sources in the 2010 reference image with poorer quality AO correction compared to the latest 2012 epoch. Taking into account that the noise increases by a factor of $\sqrt{2}$ owing to the image subtraction process, conservative $5 \sigma$ upper limits of roughly $m_{J}>19.7 \mathrm{mag}$, $m_{H}>19.9 \mathrm{mag}$, and $m_{K}>19.9 \mathrm{mag}$ were derived.

\section{Results and discussion}

The images of the nuclear and circumnuclear regions of IC 883 observed with the e-EVN and e-MERLIN are shown in Fig. 1.
In Fig. 2, we show the images resulting from a total of six VLBI epochs, covering a period of seven years. In Table 2 we report the estimated parameters for the $>5 \sigma$ sources detected in all the images.

Our new Gemini North epoch has yielded new JHK magnitude limits for SN 2011hi. In Fig. 3 we show the SN 2011hi template light curve fits from Kankare et al. (2012), including the new limits obtained from our 2012 observations for comparison.

We note that the discovery of two SNe (2010cu and 2011hi) in a $3.8 \mathrm{yr}$ period results in a $v_{\mathrm{SN}} \approx 0.5_{-0.3}^{+0.7} \mathrm{yr}^{-1}$ (using the upper and lower Poisson $1 \sigma$ uncertainties for two events given by Gehrels 1986), which agrees within the uncertainties with the IR luminosity-based CCSN rate of the galaxy. However, this estimate does not include any correction factors nor control time considerations, and should thus be regarded as a lower limit.

\subsection{The nature of SNe $2010 \mathrm{cu}$ and $2011 \mathrm{hi}$}

In Kankare et al. (2012), we investigated the nature of SNe $2010 \mathrm{cu}$ and 2011 hi by means of five epochs of NIR photometric observations prior to 2012. After comparing the light curves of SN 2010cu and SN2011hi to both the CCSN templates presented in Mattila \& Meikle (2001) and the well sampled NIR light curves of the canonical Type IIP SN 1999em (Krisciunas et al. 2009), it was concluded that the best fit for both $\mathrm{SNe}$ was either a Type IIP or a IIn/L SN (see Kankare et al. 2012). The ordinary, linearly declining template fit was 
Table 2. Estimated parameters of the detected radio-emitting sources in the (circum-)nuclear regions of IC 883.

\begin{tabular}{|c|c|c|c|c|c|c|c|c|}
\hline $\begin{array}{l}\text { Source } \\
\text { name }\end{array}$ & $\begin{array}{c}\text { Epoch } \\
\text { label }\end{array}$ & $\Delta \alpha^{s}(\mathrm{~J} 2000)$ & $\Delta \delta^{\prime \prime}(\mathrm{J} 2000)$ & $\begin{array}{c}P_{v} \\
(\mathrm{mJy} / \text { beam }) \\
\end{array}$ & $\begin{array}{c}S_{v} \\
(\mathrm{mJy})\end{array}$ & $\begin{array}{c}L_{v} \\
\left(10^{27} \mathrm{erg} \mathrm{s}^{-1} \mathrm{~Hz}^{-1}\right) \\
\end{array}$ & $\begin{array}{c}\Theta_{\mathrm{M}} \times \Theta_{\mathrm{m}} \\
\left(\mathrm{mas}^{2}\right)\end{array}$ & $\begin{array}{c}\log T_{\mathrm{B}} \\
(\mathrm{K})\end{array}$ \\
\hline (1) & (2) & (3) & (4) & (5) & (6) & (7) & (8) & (9) \\
\hline A & EM & $35.319(<1)$ & $22.35(1)$ & $4.89 \pm 0.25$ & $6.07 \pm 0.31$ & $72.65 \pm 3.67$ & $104.8 \times 30.4$ & $4.15 \pm 0.02$ \\
\hline B1a & EM & $35.332(1)$ & $22.15(3)$ & $1.04 \pm 0.07$ & $2.07 \pm 0.11$ & $24.75 \pm 1.34$ & $138.1 \times 97.1$ & $3.45 \pm 0.02$ \\
\hline B1b & EM & $35.304(1)$ & $22.63(6)$ & $0.59 \pm 0.05$ & $1.75 \pm 0.10$ & $20.97 \pm 1.17$ & $191.3 \times 141.6$ & $3.09 \pm 0.02$ \\
\hline B2a & EM & $35.356(1)$ & $21.74(3)$ & $1.03 \pm 0.07$ & $2.54 \pm 0.13$ & $30.34 \pm 1.61$ & $224.0 \times 90.5$ & $3.11 \pm 0.02$ \\
\hline $\mathrm{B} 2 \mathrm{~b}$ & EM & $35.281(2)$ & $23.05(11)$ & $0.31 \pm 0.05$ & $0.97 \pm 0.07$ & $11.55 \pm 0.78$ & $295.9 \times 109.8$ & $2.45 \pm 0.03$ \\
\hline \multirow[t]{6}{*}{$\mathrm{A} 1$} & V1 & $31.3180(\sim 0.0)$ & $22.352(0.1)$ & $1.68 \pm 0.12$ & $1.94 \pm 0.13$ & $23.19 \pm 1.54$ & $1.2 \times 0.6$ & $7.81 \pm 0.03$ \\
\hline & V2 & $31.3179(0.1)$ & $22.352(\sim 0.0)$ & $1.53 \pm 0.14$ & $1.53 \pm 0.14$ & $18.33 \pm 1.69$ & $0.6 \times \cdots$ & $6.79 \pm 0.04$ \\
\hline & V3 & $35.3181(0.1)$ & $22.349(0.1)$ & $1.95 \pm 0.15$ & $2.65 \pm 0.17$ & $31.73 \pm 2.04$ & $1.8 \times 0.3$ & $7.59 \pm 0.03$ \\
\hline & V4 & $35.3180(0.6)$ & $22.351(\sim 0.0)$ & $1.12 \pm 0.17$ & $1.21 \pm 0.17$ & $14.49 \pm 2.07$ & $13.0 \times \cdots$ & $5.97 \pm 0.06$ \\
\hline & V5 & $35.3179(0.1)$ & $22.352(\sim 0.0)$ & $3.82 \pm 0.20$ & $3.82 \pm 0.20$ & $45.67 \pm 2.42$ & $0.8 \times \cdots$ & $6.35 \pm 0.02$ \\
\hline & V6 & $35.3178(\sim 0.0)$ & $22.351(0.1)$ & $2.85 \pm 0.22$ & $4.38 \pm 0.28$ & $52.34 \pm 3.34$ & $1.5 \times 0.4$ & $7.55 \pm 0.03$ \\
\hline A2 & V5 & $35.3190(0.7)$ & $22.317(0.2)$ & $0.42 \pm 0.07$ & $0.56 \pm 0.07$ & $6.66 \pm 0.86$ & $7.0 \times 2.0$ & $5.75 \pm 0.06$ \\
\hline A3 & V6 & $35.3176(\sim 0.0)$ & $22.354(0.2)$ & $0.97 \pm 0.18$ & $1.55 \pm 0.19$ & $18.54 \pm 2.28$ & $1.4 \times 1.1$ & $7.15 \pm 0.05$ \\
\hline \multirow[t]{2}{*}{$\overline{\mathrm{A} 4}$} & V4 & $35.3181(0.7)$ & $22.371(0.1)$ & $0.89 \pm 0.17$ & $1.27 \pm 0.17$ & $15.25 \pm 2.08$ & $8.1 \times 0.6$ & $5.98 \pm 0.06$ \\
\hline & V5 & $35.3187(0.9)$ & $22.366(0.2)$ & $0.33 \pm 0.07$ & $0.67 \pm 0.07$ & $8.06 \pm 0.89$ & $14.0 \times 1.4$ & $5.23 \pm 0.05$ \\
\hline
\end{tabular}

Notes. Columns: (1) Source names corresponding to those in Figs. 1 and 2. (2) Epoch label corresponding to those specified in Table 1. Note that epoch EM and V5 are quasi-simultaneous. (3-4) Coordinates given with respect to $\alpha(J 2000)=13^{\mathrm{h}} 20^{\mathrm{m}} 00^{\mathrm{s}} 0$ and $\delta(J 2000)=34^{\circ} 08^{\prime} 00{ }^{\prime} 0$. Position uncertainties in mas, within parentheses, are given in terms of the full width at half maximum (FWHM) by $F W H M /(2 \times \mathrm{S} / \mathrm{N})$, where $\mathrm{S} / \mathrm{N}$ is the signal-to-noise ratio, and FWHM was taken as the projection of the beam major axis on both $\alpha$ and $\delta$ axes. (5) Peak intensity. We estimated the uncertainties by adding in quadrature the rms noise in the map (Col. 10 in Table 1) to a $5 \%$ uncertainty in the point-source flux density calibration. (6) Flux density. (7) Monochromatic luminosity at the observed frequency (Col. 5 in Table 1). (8) Deconvolved major and minor axes, obtained by fitting a Gaussian to each source. (9) Brightness temperature. When no deconvolved sizes could be obtained, we instead used the solid angle subtended by the synthesised beam for calculating lower limits of $T_{\mathrm{B}}$, i.e., $\Omega_{\mathrm{s}}=\pi(4 \log 2)^{-1}\left(F W H M_{\mathrm{M}} \times F W H M_{\mathrm{m}}\right)$, where $F W H M_{\mathrm{M}}$ and $F W H M_{\mathrm{m}}$ are the major and minor synthesised beam-fitted FWHM (Col. 9 in Table 1), respectively.
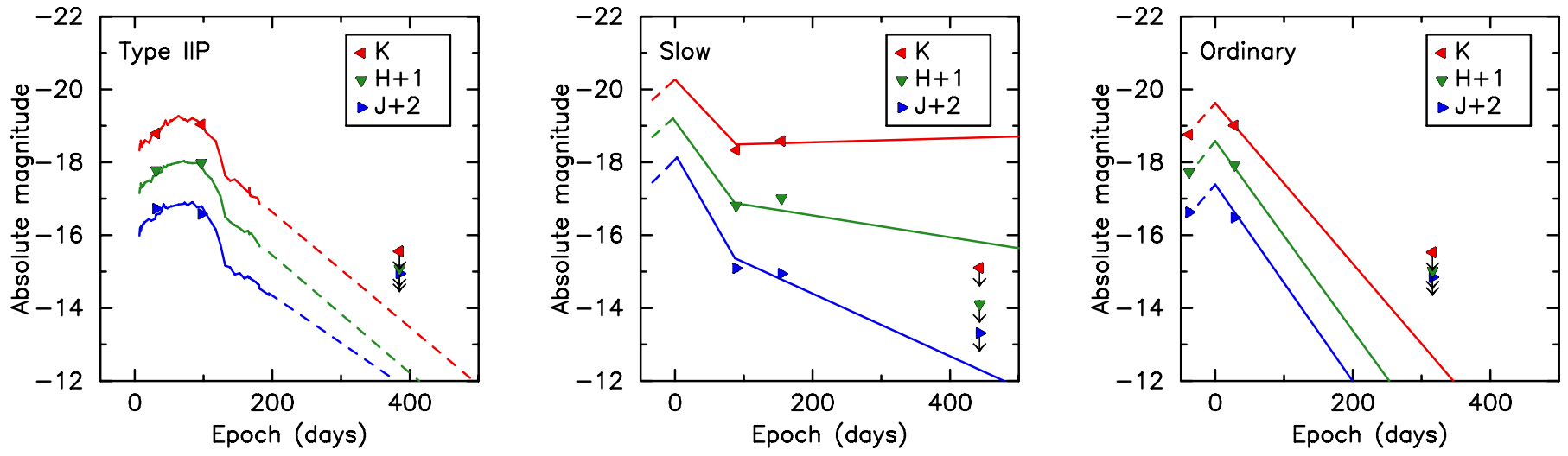

Fig. 3. Template light-curve fits for SN2011hi presented in Kankare et al. (2012) adopting the Calzetti et al. (2000) extinction law, with the addition of the late-time $J H K$ upper limits obtained for SN2011hi in this work. The epochs not covered by the templates are linearly extrapolated and marked with the dashed lines.

previously excluded because this fit corresponds to an extremely early discovery and an unrealistically long rise time to the light curve peak. A NIR-bright Type IIn/L SN with $\sim 0$ mag of extinction was proposed by Kankare et al. (2012) as the type of SN 2011hi due to the low $(<2 \mathrm{mag})$ total line-of-sight extinction implied by the $J-K$ colour map of the galaxy. However, a Type IIP light curve with higher extinction could also provide a good fit to the data, and as noted in Kankare et al. (2012), higher localized extinctions are possible, as the colour map is tracing only the large scale structures of the LIRG.

In Fig. 3 we present the template $\chi^{2}$ fits of the SN 2011hi light curves adopting the Calzetti et al. (2000) reddening law and including the new upper limits. However, these limits are not included in the fit, i.e., the values reported in the Table 2 of Kankare et al. (2012) are unchanged. Based on the new data, the slowly declining (NIR-bright Type IIn/L) SN template is now excluded due to the fading of the SN. In addition to the SN 1999em template, we have now also compared the NIR light curve of SN 2011hi with that of other Type IIP SNe: 2003hn (Krisciunas et al. 2009) and 2004et (Maguire et al. 2010). The results of the best fits are similar to those obtained with SN 1999em, but have larger $\chi^{2}$ values. The $V$-band extinctions inferred for the three different Type IIP SNe used for comparison are consistent within $0.5 \mathrm{mag}$ for the adopted extinction law. We conclude that SN 2011hi was a slightly over-luminous (1-2 mag brighter than SN 1999em) Type IIP SN discovered roughly a month after its SN explosion, with 5 or $7 \mathrm{mag}$ of host galaxy extinction in the $V$-band when adopting either the Cardelli et al. (1989) or the Calzetti et al. (2000) reddening law, respectively.

We note that no radio sources were detected with the e-EVN with confidences higher than $3 \sigma$ at/around the reported positions for either SN2010cu, or SN 2011hi. Therefore, the 
$3 \sigma$ upper limits at $5 \mathrm{GHz}$ correspond to $2.4 \times 10^{27} \mathrm{erg} \mathrm{s}^{-1} \mathrm{~Hz}^{-1}$ for both $\mathrm{SNe}$. The $\mathrm{SNe}$ were also not detected in the archival 8.4 GHz VLBA observations (project BC196) carried out two months after our e-EVN observations. Source B2a detected with e-MERLIN at $6.9 \mathrm{GHz}$ is positionally consistent within the uncertainties with SN 2010cu. However, since B2a is not a compact source, we discard the possibility of it being a SN.

SN 2010cu, had an estimated age of 14-17 months at the time of the radio observations made in 2011. Its non-detection at $5,6.9$, and $8.4 \mathrm{GHz}$ is an indication that: (i) its radio emission at these frequencies has entered the optically thin decline phase of its evolution (e.g., a Type Ib/c SN), with a luminosity well below $2.4 \times 10^{27} \mathrm{erg} \mathrm{s}^{-1} \mathrm{~Hz}^{-1}$; and/or (ii) SN 2010cu is an intrinsically low-luminosity Type II radio supernova (e.g., a Type IIP such as SN 1999em) and thus more sensitive observations would be required for its detection.

SN2011hi was also undetected in our radio observations made 2-5 months after its explosion, which is consistent with Type IIP SNe being low-luminosity radio emitters. Other well-known type IIP SNe such as SNe 1999em, 2004dj, and 2004et (Pooley et al. 2002; Beswick et al. 2005; Martí-Vidal et al. 2007, respectively) have peak radio luminosities below $10^{26} \mathrm{erg} \mathrm{s}^{-1} \mathrm{~Hz}^{-1}$ (see e.g., Fig. 2 in Chevalier et al. 2006). At NIR wavelengths, SN 2011hi was only slightly brighter than $\mathrm{SN} 1999 \mathrm{em}$. If its radio emission were likewise only slightly stronger, we would expect its radio luminosity to be well below the $3 \sigma$ detection threshold $\left(2.4 \times 10^{27} \mathrm{erg} \mathrm{s}^{-1} \mathrm{~Hz}^{-1}\right)$ of our most sensitive radio observations carried out with the e-EVN. We note that the non-detection of SNe 2010cu and 2011hi at radio wavelengths does not imply that no radio emission has been produced by these NIR-detected SNe.

To gain a clearer understanding of SNe in LIRGs, it is desirable to perform a radio monitoring of the NIR-detected $\mathrm{SNe}$ by including observations at different frequencies over many epochs. This strategy would help us to detect either a rapidly or slowly evolving SN, which would also help to constrain the SN type (Stockdale et al. 2007). However, not all CCSNe will be bright radio emitters. This depends on the interaction between the SN ejecta and the circumstellar medium, which gives rise to the synchrotron emission at radio frequencies (see e.g., Chevalier 1982).

\subsection{The circumnuclear regions of IC 883: a jet or a disc?}

The quest to detect radio emission from SNe 2010cu and 2011hi has enabled us to unveil the different facets of IC 883. In the following, we discuss the circumnuclear structure of IC 883 and the nature of its nuclear regions.

Our e-MERLIN observations reveal a double-sided structure with an approximate projected extent of $2 \operatorname{arcsec}(\sim 1 \mathrm{kpc})$ by $0.3 \operatorname{arcsec}(\approx 150 \mathrm{pc})$, at a position angle of $130^{\circ}$ (Fig. 1). This inclination matches that previously found in other radio studies (e.g., Condon et al. 1991; Parra et al. 2010). However, the double-sided structure in IC 883 is revealed for the first time at $6.9 \mathrm{GHz}$ with our e-MERLIN observations (see also the $1.4 \mathrm{GHz}$ MERLIN map from Clemens \& Alexander 2004). VLA observations (e.g., from Condon et al. 1991; Parra et al. 2010) are of insufficient spatial resolution to image the circumnuclear regions with a similar level of detail. By convolving our eMERLIN image with a beam of size 0.4 , which corresponds to VLA A-configuration observations at $5 \mathrm{GHz}$, we were able to recover a structure similar to that shown in Fig. 2 of Parra et al. (2010).
At first glance, the structure seen with e-MERLIN resembles that of a two-sided jet of an AGN, whose restarting activity explains the presence of two sets of condensations (B1a-B1b and B2a-B2b; see left panel in Fig. 1), at either side of the core A. The higher flux density of the approaching components in the jet, which are $\mathrm{B} 1 \mathrm{a}$ and $\mathrm{B} 2 \mathrm{a}$ in this case, with respect to the flux density of the receding components, $\mathrm{B} 1 \mathrm{~b}$ and $\mathrm{B} 2 \mathrm{~b}$, could be explained in terms of Doppler boosting effects.

There are however strong arguments against a jet interpretation: (i) the approaching components are at shorter distances from the core than the receding components, thus implying that their apparent velocities are smaller; (ii) the putative jet structure is placed on top of the disc of the galaxy, which is difficult to explain, unless we consider that the directions of jet and disc coincide owing to a projection effect.

Different studies of the molecular and atomic gas towards IC 883 (Downes \& Solomon 1998; Bryant \& Scoville 1999; Clemens \& Alexander 2004) provide evidence of the rotation of the disc from south-east towards north-west. This situation would imply that the putative jet is perpendicular to the rotation axis, and hence that no projection effect could explain the superposition of the jet on the disc. Schmitt et al. (2002) studied the orientation of jets with respect to the rotation axis in a sample of radio galaxies and found that large misalignments $\left(<77^{\circ}\right)$ may occur because of warping mechanisms acting in the black hole's accretion disc. In the case of IC 883, the misalignment would be close to $90^{\circ}$, so a jet scenario for the structure revealed with e-MERLIN is even less viable.

If we consider instead a rotating disc (or ring) scenario, component A would be at the centre, while B1a and B2a (approaching components) and $\mathrm{B} 1 \mathrm{~b}$ and $\mathrm{B} 2 \mathrm{~b}$ (receding components) would be continuum radio components of the disc itself. We note that the line joining component B1a with B1b through $\mathrm{A}$, is not the same as the line joining B2a with B2b, which provides evidence of the disc/ring being warped (in agreement with previous studies, e.g., Clemens \& Alexander 2004), probably due to the interaction with the ambient material. We note that the structure unveiled by e-MERLIN has a width of approximately $150 \mathrm{pc}$. Whilst this width might appear to be too narrow for representing a disc, thin discs of a few hundreds of parsecs are not uncommon for edge-on galaxies at radio frequencies (Dumke et al. 1995). Furthermore, e-MERLIN resolves out some of the extended emission that is otherwise visible at lower resolution, for instance with the VLA (Condon et al. 1991; Parra et al. 2010), and hence, the size of the structure mapped with e-MERLIN is compatible with a disc/ring structure.

Thus, whilst we cannot completely rule out that the structure mapped by e-MERLIN is a jet, there is strong evidence both against this, and it may instead be a rotating disc/ring.

Further e-MERLIN observations at different frequencies could be used to probe the radio lifetime of the condensations on either side of the core A. Observations optimized for polarimetry studies would yield information on the configuration of the large-scale magnetic field, which could probe the existence of shocked material as expected in a jet scenario, hence helping us to arrive to a firm conclusion.

\subsection{The ongoing nuclear activity in IC 883}

Our e-EVN observations (see right panel in Fig. 1), performed simultaneously with our e-MERLIN observations, reveal at least two compact sources (labelled $\mathrm{A} 1$ and $\mathrm{A} 2$ ) above $5 \sigma$ in the innermost nuclear regions of IC 883. The high brightness 
temperatures of sources A1 and A2 are both indicative of a nonthermal origin.

The brightest component in our e-MERLIN image (A) has a peak intensity similar to that of A1. By adding up the flux densities of components A1 and A2 in our e-EVN map, we recover a flux density of $4.38 \pm 0.27 \mathrm{mJy}$, which is still lower than both the peak intensity and the flux density of component A (see Table 2). We find that A1 provides a strong contribution to the flux density on larger scales (although seen at slightly different frequencies), and furthermore, has a position highly coincident with component $\mathrm{A}$, despite the use of different phase reference sources and different spatial resolutions. Hence, A1 is very likely the main source producing the radio emission of component A.

From our e-EVN observations alone, we can neither characterise the natures of sources A1 and A2, nor investigate a possible relation between components $\mathrm{A} 1$ and $\mathrm{A}$. We therefore searched for additional, publicly available, archival VLBI data (see Table 1) that could be of use to investigate these issues.

Component A1 from our e-EVN observations is detected in all the additional VLBI observations (see Fig. 2) and appears to fluctuate in terms of flux density at $5 \mathrm{GHz}$, varying by at least a factor of three between the minimum and maximum values displayed within the seven years spanning the different VLBI epochs (see Table 2). Component A2 is only detected in experiment RR006. At $8.4 \mathrm{GHz}$ (experiment BC196), we detect a $5 \sigma$ source (A3) north-west of component A1. The resolution of our e-EVN observations (RR006) does not allow us to detect component A3, but the source is also undetected in the VLBA epochs at $5 \mathrm{GHz}$, whose data have a resolution similar to that of experiment BC196. A $3 \sigma$ component located north-east of component $\mathrm{A} 1$, is detected as a $5 \sigma$ source in experiment EP055 and labelled A4. This source has no counterpart at $8.4 \mathrm{GHz}$ in the observations of project $\mathrm{BC} 196$, indicating that at that frequency the emission from A4 has become optically thin, as expected in a $\mathrm{SN}$ scenario. We thus have a total of three transient sources (A2, $\mathrm{A} 3$, and A4) in the nucleus of IC 883, in addition to the more luminous, variable, component $\mathrm{A} 1$, which has remained visible for at least seven years.

\subsection{The nature of the compact sources in the IC 883 nucleus}

The milliarcsec sources detected in the innermost regions of IC 883 are evidence of its ongoing nuclear activity. A variable source, A1, was detected in six different VLBI epochs (see Table 2 and Fig. 2) over seven years, and appears to be the main powering source of the emission on the larger scales imaged with e-MERLIN. Three transient sources were also detected. All the sources have sizes and brightness temperatures that correspond to a non-thermal origin. The transient sources are most probably $\mathrm{SNe}$ and/or SN remnants (SNR), whereas for component A1 the nature is less clear. To investigate the nature of sources A1-A4, we compare their luminosities with those reported in the literature for the non-thermal sources (SNe and SNRs) at $5 \mathrm{GHz}$ in the starburst galaxy M 82, the LIRG Arp 299-A, and in the ULIRG Arp 220 (Fig. 4).

From Fig. 4, we see that the nuclear components of IC 883, although less numerous, are in general more luminous than the components identified as SNe and SNRs in the nuclear regions of other galaxies with high star formation rates. The lifetime of the transient sources in IC 883 ranges from less than a few months up to a few years. It is thus very likely that these sources are a mixture of SNe and SNRs, which therefore implies that there has been a recent starburst activity in the nucleus of IC 883 . We lack

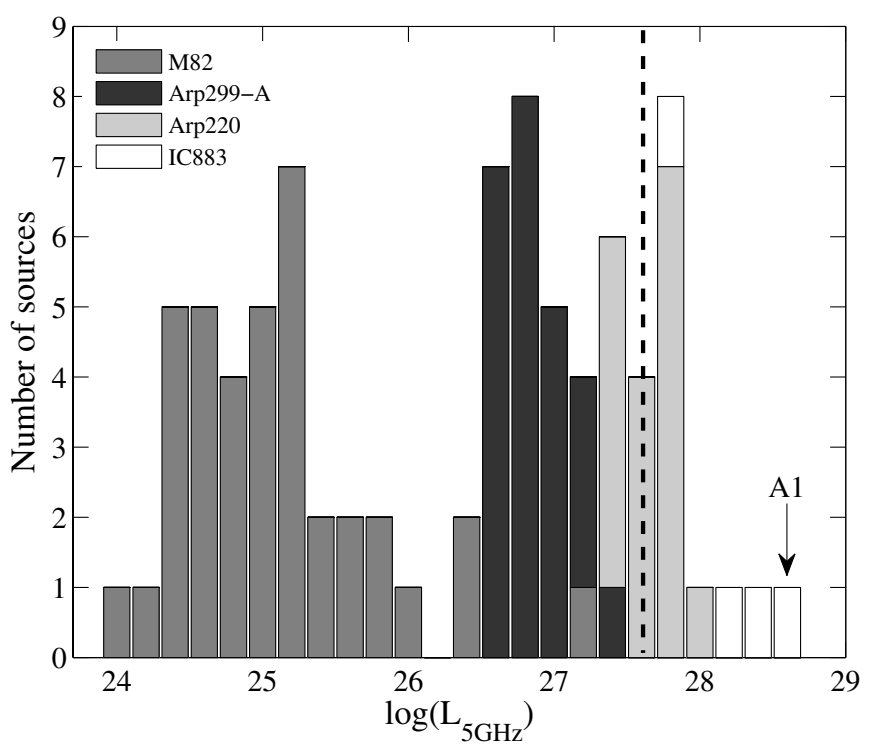

Fig. 4. Luminosity distribution at $5 \mathrm{GHz}$ of $\mathrm{SNe}$ and $\mathrm{SNRs}$ in $\mathrm{M} 82$, Arp 299-A, and Arp 220. All the IC 883 nuclear sources have been added for comparison purposes. Each bin has a width of $\log _{10}\left(L_{5 \mathrm{GHz}}\right)=$ 0.2 with $L_{5 \mathrm{GHz}}$ in units of $\mathrm{erg} \mathrm{s}^{-1} \mathrm{~Hz}^{-1}$. The SN and SNR luminosities have been retrieved from Fenech et al. (2008) and Marchili et al. (2010) for M 82, from Bondi et al. (2012) for Arp 299-A, and from Batejat et al. (2011) for Arp 220. The most luminous M 82 source corresponds to SN 2008iz. In the case of Arp 220, we have included SNe and SNRs from both the west and east nuclei. The luminosities of the IC 883 components were taken from Table 2: from experiment RR006 for $\mathrm{A} 1$ and $\mathrm{A} 2$, from experiment $\mathrm{BC} 196$ for $\mathrm{A} 3$, and from experiment EP055 for A4. We have assumed a spectral index of -0.7 to obtain the luminosity at $5 \mathrm{GHz}$ of component $\mathrm{A} 3$. Note that $\mathrm{A} 1$ is the brightest source, and with our most sensitive VLBI image (RR006) we are only sensitive to sources above a $5 \sigma$ luminosity limit of $4 \times 10^{27} \mathrm{erg} \mathrm{s}^{-1} \mathrm{~Hz}^{-1}$, which is indicated by a dashed line in the histogram.

the spectral information that might have helped to differentiate between a SN or a SNR origin for each of these transient sources.

Component A1 is the most luminous of all the sources that we have studied (see histogram in Fig. 4). A SN or a SNR origin are not very likely, based on its flux density fluctuations (see Cols. 5 and 6 in Table 2), its longevity, and its relatively flat two-point spectral index between 5 and $8.4 \mathrm{GHz}, \alpha=0.3$ $\left(S_{v} \sim v^{\alpha}\right)$, calculated from the flux densities obtained in experiments RR006 and BC196. Therefore, A1 is very likely an AGN.

Further evidence of an obscured AGN in IC 883 can be inferred from the ratio of radio to hard X-ray luminosity $\left(R_{\mathrm{X}}=\right.$ $v L_{v}(5 \mathrm{GHz}) / L_{\mathrm{HX}}$; Terashima \& Wilson 2003). Iwasawa et al. (2011) report a hard X-ray luminosity $\left(L_{\mathrm{HX}}=L[2-10 \mathrm{keV}]\right)$ of $6.4 \times 10^{40} \mathrm{erg} \mathrm{s}^{-1}$ for IC 883 . Considering the luminosity at $5 \mathrm{GHz}$ of A1 observed with the e-EVN (see Table 2), we calculate $R_{\mathrm{X}} \approx 3.6 \times 10^{-3}$, which is consistent with a low-luminosity AGN (LLAGN) or a normal AGN in a radio galaxy, as inferred from Fig. 4 of Terashima \& Wilson (2003).

By comparing the maximum variability (a factor of about three) displayed by A1 in our observations, with the largest inter-year variability displayed by typical LLAGNs (Nagar et al. 2002), we find that A1 would be placed at the high end of the LLAGN behaviour. Thus, we can only conclude that A1 is a candidate to be either a normal AGN or a low-luminosity one.

Component A4 whose elongation could be a sort of outflow, has however no counterpart at $8.4 \mathrm{GHz}$ (experiment BC196). Future VLBI observations at different frequencies should allow an unequivocal characterisation of the AGN candidate 


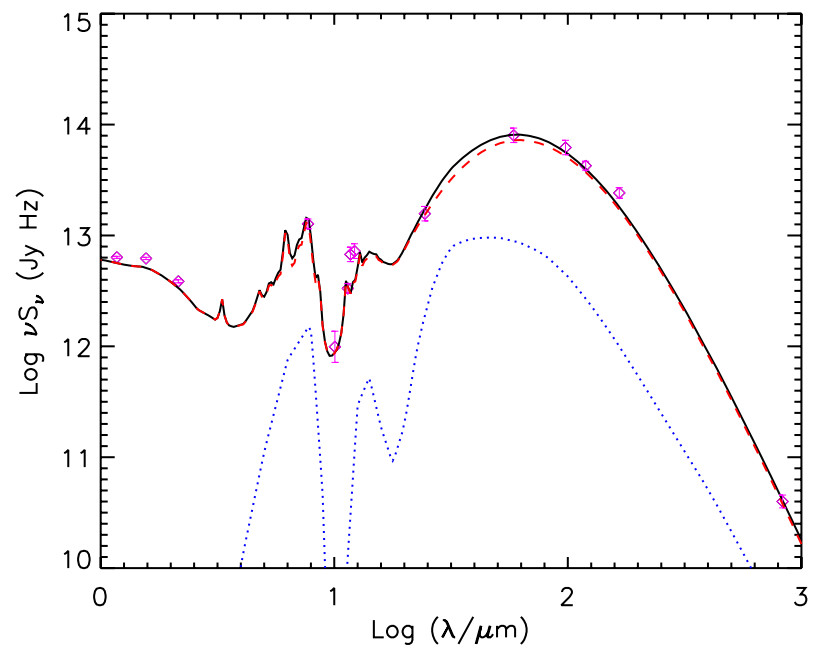

Fig. 5. The infrared SED of IC 883 including 2MASS and IRAS data, as well as the SCUBA point from Dunne et al. (2000). The red-dashed line is our starburst model fit, and the blue-dotted line is the model fit for an AGN upper limit contribution. The black-solid line is the model fit including both components.

in IC 883, as well as the possible discovery of additional nuclear components.

If we considered sources A2, A3, and A4 to have an origin in recent star formation activity, we would have three CCSN events that occurred in the period between June 2006 and May 2011, i.e., five years. We can thus estimate a CCSN rate for the IC 883 nuclear region of $\approx 0.6_{-0.3}^{+0.6} \mathrm{yr}^{-1}$, using the upper and lower Poisson $1 \sigma$ uncertainties for three events given by Gehrels (1986). Combining the nuclear CCSN rate with the CCSN rate inferred from the detection of SNe 2010cu and 2011hi within $3.8 \mathrm{yr}$ in the circumnuclear regions of IC 883, we obtain a CCSN rate for the entire galaxy of $\approx 1.1_{-0.6}^{+1.3} \mathrm{yr}^{-1}$. These rates are considered as lower limits since our irregular temporal sampling at both radio and NIR wavelengths might have missed rapidly evolving $\mathrm{SNe}$, and also because our $5 \sigma$ radio luminosity detection threshold $\left(4 \times 10^{27} \mathrm{erg} \mathrm{s}^{-1} \mathrm{~Hz}^{-1}\right)$ prevents us from detecting Type IIP and IIb SNe, and even some Type IIL. We note that the total rate estimate agrees with the IR luminosity-based CCSN rate estimate (see Sect. 1). It is thus of great interest to estimate the contribution of a putative AGN to the IR luminosity in order to obtain a more reliable estimate of the expected CCSN rate.

\subsection{The infrared SED of IC 883: estimating the contribution of an AGN}

As mentioned in Sect. 1, IC 883 was classified by Yuan et al. (2010) as a composite starburst-AGN, based on its optical diagnostic diagrams.

We modelled the spectral energy distribution (SED) of IC 883 (see Fig. 5) to estimate the contribution of an AGN to the total IR luminosity and provide an estimate of the CCSN rate in this galaxy. For this, we used a grid of AGN dusty torus models that were computed with the method of Efstathiou \& Rowan-Robinson (1995) and a grid of starburst models that were computed with the method of Efstathiou et al. (2000), but with a revised dust model (Efstathiou \& Siebenmorgen 2009). The spatial resolution of the mid- and far-IR data used for the modelling is not high enough to spatially separate the different components.
Therefore, to distinguish the contribution of the AGN torus from the starburst we fitted a model combining both an AGN and a starburst.

For the AGN, we considered a grid of tapered disc models computed with the method of Efstathiou \& Rowan-Robinson (1995). In this grid of models, we considered four discrete values for the equatorial $1000 \AA$ optical depth $(500,750,1000,1250)$, three values for the ratio of outer to inner disc radii $(20,60$, $100)$, and three values for the opening angle of the disc $\left(30^{\circ}\right.$, $45^{\circ}$ and $60^{\circ}$ ). The best-fit model parameters corresponding to the minimum $\chi^{2}$ when we combine the starburst and the AGN torus models, are an opening angle of $60^{\circ}$ for the torus, an equatorial optical depth of 1250 at $1000 \AA$, and a ratio of outer to inner radius of 60 . The AGN torus is viewed close to edge-on owing to the IC 883 geometry; thus, its apparent contribution to the total infrared emission is insignificant (probably $<10$ per cent of the starburst luminosity). Therefore, the starburst clearly dominates the observed luminosity of the entire galaxy. We note that owing to our almost edge-on view of the torus and its extremely high optical depth, the intrinsic luminosity of the AGN itself could be a factor of about 30 times higher than the observed one. This enhancing factor is related to the anisotropy of the torus emission (see Efstathiou 2006, for a definition), and implies that the intrinsic luminosity of the AGN could be about three times higher than the observed starburst luminosity.

Our model incorporates the stellar population synthesis model of Bruzual \& Charlot (2003), which makes a prediction of the $\mathrm{SN}$ rate, $v_{\mathrm{SN}}(t)$, at a time $t$ after star formation in an instantaneous burst. The starburst model of Efstathiou et al. (2000) predicts the spectrum of this instantaneous burst at time $t$ and assumes a star formation history for the starburst. We assumed that the SFR in IC 883 declines exponentially with an e-folding time of $20 \mathrm{Myr}$, and that the age of the starburst is $55 \mathrm{Myr}$. The initial optical depth of the molecular clouds that constitute the starburst is assumed to be 100 . The SFR averaged over the duration of the starburst is $185 M_{\odot} \mathrm{yr}^{-1}$. It is also possible to calculate self-consistently the $\mathrm{SN}$ rate at different stages of the starburst evolution by convolving the star formation history with $v_{\mathrm{SN}}(t)$. This yields a $v_{\mathrm{SN}}$ of $1.1 \mathrm{yr}^{-1}$, which is relevant to the entire galaxy and can therefore be compared with the observed values. We combined the CCSN rate for the nuclear regions, which is based on three radio SNe detected in our VLBI observations, with the CCSN rate for the circumnuclear regions based on the discovery of SNe 2010cu and 2011hi, which resulted in a lower limit to the CCSN rate of $\approx 1.1_{-0.6}^{+1.3} \mathrm{yr}^{-1}$ for the entire galaxy (see Sect. 3.4). This CCSN rate estimate is in good agreement with the predicted CCSN rate for the entire IC 883 galaxy from the IR SED modelling.

Although IC 883 is less luminous in the IR but farther away than Arp 299-A and in a much more advanced merger stage, it also contains both a starburst and an AGN. The nuclear starburst in IC 883 is apparently less prolific than in Arp 299-A (Pérez-Torres et al. 2009). However, the AGN in IC 883 seems to be stronger than the one in Arp 299-A (Pérez-Torres et al. 2010), and even powers the radio luminosity on circumnuclear scales, although its contribution to the emission of the entire galaxy at IR wavelengths is apparently insignificant.

\section{Summary}

We have imaged the nuclear and circumnuclear regions of the LIRG IC 883 at radio frequencies. Our e-EVN observations, together with archival VLBI observations, reveal the presence 
of at least four non-thermal components in the nuclear regions of IC 883. Three of these are transient sources and one is a longlived, variable compact source, which is very likely an AGN. Our e-MERLIN observations show a striking double-sided structure, which likely represents a warped disc/ring, in agreement with previous studies on the interstellar medium of this galaxy.

The source we have identified as an AGN candidate powers the radio emission in IC 883 on nuclear and circumnuclear scales, and yet, as indicated by our radio and NIR observations, and our model of the IR SED, this LIRG displays very active star formation both within the innermost $100 \mathrm{pc}$ of its nucleus, and the circumnuclear regions. We have estimated lower limits to the CCSN rate in the innermost nuclear regions of $0.6_{-0.3}^{+0.6} \mathrm{yr}^{-1}$ based on the detection of three radio $\mathrm{SNe}$ above a $5 \sigma$ luminosity threshold of $4 \times 10^{27} \mathrm{erg} \mathrm{s}^{-1} \mathrm{~Hz}^{-1}$, and $1.1_{-0.6}^{+1.3} \mathrm{yr}^{-1}$ for the entire galaxy, based on the nuclear radio SNe and the NIR SNe 2010cu and $2011 \mathrm{hi}$ discovered in the circumnuclear regions. Our most recent NIR Gemini North observations of IC 883 clarify the nature of SN 2011hi as a Type IIP SN, in agreement with its nondetection in our observations at radio frequencies.

The composite starburst-AGN nature of IC 883, revealed previously by optical spectroscopy (e.g., Yuan et al. 2010), is now supported by our radio observations for the first time, thus making IC 883 one of the few galaxies where the starburst-AGN connection can be studied in detail.

Acknowledgements. We thank the anonymous referee for his/her comments. The authors are thankful to the EVN directors for their rapid provision of targetof-opportunity observations. The European VLBI Network is a joint facility of European, Chinese, South African, and other radio astronomy institutes funded by their national research councils. We also made use of observations with e-MERLIN, the UK's facility for high resolution radio astronomy observations, operated by The University of Manchester for the Science and Technology Facilities Council; and observations obtained at the Gemini Observatory, which is operated by the Association of Universities for Research in Astronomy, Inc., under a cooperative agreement with the NSF on behalf of the Gemini partnership: the National Science Foundation (US), the Science and Technology Facilities Council (UK), the National Research Council (Canada), CONICYT (Chile), the Australian Research Council (Australia), Ministério da Ciência e Tecnologia (Brazil), and Ministerio de Ciencia, Tecnología e Innovación Productiva (Argentina). This article is also based on observations made with the Very Long Baseline Array (VLBA) of the National Radio Astronomy Observatory (NRAO); the NRAO is a facility of the National Science Foundation operated under cooperative agreement by Associated Universities, Inc. This work made use of the Swinburne University of Technology software correlator, developed as part of the Australian Major National Research Facilities Programme and operated under licence. Our work is supported by the European Community Framework Programme 7, Advanced Radio Astronomy in Europe, grant agreement No. 227290. C.R.-C., M.A.P.-T. and A.A. acknowledge financial support from the Spanish MICINN through grant AYA2009-13036-C02-01, co-funded with FEDER funds. S.M., E.K. and C.R.-C. acknowledge financial support from the Academy of Finland (project: 8120503). E.K. acknowledges support from the Finnish Academy of Science and Letters (Vilho, Yrjö and Kalle Väisälä Foundation).

\section{References}

Alard, C. 2000, A\&AS, 144, 363

Alard, C., \& Lupton, R. H. 1998, ApJ, 503, 325
Batejat, F., Conway, J. E., Hurley, R., et al. 2011, ApJ, 740, 95

Beswick, R. J., Muxlow, T. W. B., Argo, M. K., et al. 2005, ApJ, 623, L21

Bondi, M., Pérez-Torres, M. A., Herrero-Illana, R., \& Alberdi, A. 2012, A\&A, 539, A134

Bruzual, G., \& Charlot, S. 2003, MNRAS, 344, 1000

Bryant, P. M., \& Scoville, N. Z. 1999, AJ, 117, 2632

Calzetti, D., Armus, L., Bohlin, R. C., et al. 2000, ApJ, 533, 682

Cardelli, J. A., Clayton, G. C., \& Mathis, J. S. 1989, ApJ, 345, 245

Chevalier, R. A. 1982, ApJ, 259, 302

Chevalier, R. A., Fransson, C., \& Nymark, T. K. 2006, ApJ, 641, 1029

Clemens, M. S., \& Alexander, P. 2004, MNRAS, 350, 66

Condon, J. J., Huang, Z.-P., Yin, Q. F., \& Thuan, T. X. 1991, ApJ, 378, 65

Deller, A. T., Brisken, W. F., Phillips, C. J., et al. 2011, PASP, 123, 275

Downes, D., \& Solomon, P. M. 1998, ApJ, 507, 615

Dumke, M., Krause, M., Wielebinski, R., \& Klein, U. 1995, A\&A, 302, 691

Dunne, L., Eales, S., Edmunds, M., et al. 2000, MNRAS, 315, 115

Efstathiou, A. 2006, MNRAS, 371, L70

Efstathiou, A., \& Rowan-Robinson, M. 1995, MNRAS, 273, 649

Efstathiou, A., \& Siebenmorgen, R. 2009, A\&A, 502, 541

Efstathiou, A., Rowan-Robinson, M., \& Siebenmorgen, R. 2000, MNRAS, 313, 734

Fenech, D. M., Muxlow, T. W. B., Beswick, R. J., Pedlar, A., \& Argo, M. K. 2008, MNRAS, 391, 1384

Gehrels, N. 1986, ApJ, 303, 336

Iwasawa, K., Sanders, D. B., Teng, S. H., et al. 2011, A\&A, 529, A106

Kankare, E., Mattila, S., Ryder, S., et al. 2008, ApJ, 689, L97

Kankare, E., Ryder, S. D., Mattila, S., et al. 2011, Central Bureau Electronic Telegrams, 2889, 1

Kankare, E., Mattila, S., Ryder, S., et al. 2012, ApJ, 744, L19

Keel, W. C. 1985, AJ, 90, 2207

Kewley, L. J., Groves, B., Kauffmann, G., \& Heckman, T. 2006, MNRAS, 372, 961

Krisciunas, K., Hamuy, M., Suntzeff, N. B., et al. 2009, AJ, 137, 34

Maguire, K., Di Carlo, E., Smartt, S. J., et al. 2010, MNRAS, 404, 981

Marchili, N., Martí-Vidal, I., Brunthaler, A., et al. 2010, A\&A, 509, A47

Martí-Vidal, I., Marcaide, J. M., Alberdi, A., et al. 2007, A\&A, 470, 1071

Mattila, S., \& Meikle, W. P. S. 2001, MNRAS, 324, 325

Modica, F., Vavilkin, T., Evans, A. S., et al. 2012, AJ, 143, 16

Nagar, N. M., Falcke, H., Wilson, A. S., \& Ulvestad, J. S. 2002, A\&A, 392, 53

Parra, R., Conway, J. E., Aalto, S., et al. 2010, ApJ, 720, 555

Pérez-Torres, M. A., Romero-Cañizales, C., Alberdi, A., \& Polatidis, A. 2009. A\&A, 507, L17

Pérez-Torres, M. A., Alberdi, A., Romero-Cañizales, C., \& Bondi, M. 2010, A\&A, 519, L5

Pooley, D., Lewin, W. H. G., Fox, D. W., et al. 2002, ApJ, 572, 932

Ryder, S., Kankare, E., \& Mattila, S. 2010, Central Bureau Electronic Telegrams, 2286, 1

Sanders, D. B., \& Mirabel, I. F. 1996, ARA\&A, 34, 749

Sanders, D. B., Mazzarella, J. M., Kim, D., Surace, J. A., \& Soifer, B. T. 2003, AJ, 126, 1607

Schmitt, H. R., Pringle, J. E., Clarke, C. J., \& Kinney, A. L. 2002, ApJ, 575, 150

Scoville, N. Z., Evans, A. S., Thompson, R., et al. 2000, AJ, 119, 991

Shepherd, M. C., Pearson, T. J., \& Taylor, G. B. 1995, in BAAS 27, ed. B. J. Butler, \& D. O. Muhleman, 903

Smith, D. A., Herter, T., Haynes, M. P., Beichman, C. A., \& Gautier III, T. N. 1995, ApJ, 439, 623

Smith, H. E., Lonsdale, C. J., \& Lonsdale, C. J. 1998, ApJ, 492, 137

Stockdale, C. J., Kelley, M. T., Weiler, K. W., et al. 2007, in Supernova 1987A: 20 Years After: Supernovae and Gamma-Ray Bursters, ed. S. Immler, K. Weiler, \& R. McCray, AIP Conf. Ser., 937, 264

Terashima, Y., \& Wilson, A. S. 2003, ApJ, 583, 145

Valenti, S., Fraser, M., Benetti, S., et al. 2011, MNRAS, 416, 3138

Veilleux, S., Kim, D.-C., Sanders, D. B., Mazzarella, J. M., \& Soifer, B. T. 1995, ApJS, 98, 171

Yuan, T.-T., Kewley, L. J., \& Sanders, D. B. 2010, ApJ, 709, 884 